\title{
QUE IMPORTÂNCIA ATRIBUEM OS ESTUDANTES DE ENFERMAGEM ÀS NOVAS TECNOLOGIAS
}

\author{
Maria do Céu Sá1 e Ana Sofia Nabais ${ }^{2}$ \\ 1Escola Superior de Enfermagem de Lisboa (ESEL), UI\&DE Portugal. ceu.sa@esel.pt \\ ESEL, Centro Hospitalar Universitário Lisboa Norte, Portugal. ana.nabais@esel.pt.
}

\begin{abstract}
Resumo. O desenvolvimento da ciência e da tecnologia introduziu mudanças significativas na área de saúde, constituindo um desafio para a prestação dos cuidados de enfermagem. Na formação dos enfermeiros, vários tipos de equipamentos e tecnologias educacionais são utilizados salientando-se as tecnologias de informação e comunicação (TIC), cujo objetivo é facilitar o processo de ensinoaprendizagem, complementando os métodos de ensino tradicionais. As escolas de enfermagem têm por objetivo preparar futuros enfermeiros habilitados a utilizar as novas tecnologias emergentes e trabalhar em contextos diversificados. Neste artigo, pretendeu-se compreender a importância que os estudantes de enfermagem atribuem às TIC no processo de formação. A metodologia adotada foi a realização de entrevistas individuais com estudantes de enfermagem, no tratamento de dados utilizou-se a análise de conteúdo. Os resultados revelaram que os estudantes reconhecem as vantagens das TIC na sua aprendizagem como futuros profissionais de saúde. Estes usam principalmente o computador e telemóvel com o objetivo de realizar pesquisa, comunicar e efetuar estudo autónomo, o que facilita o processo de ensino-aprendizagem, a tomada de decisão e a promoção da continuidade nos cuidados de enfermagem. Os estudantes de enfermagem consideram as TIC como ferramentas facilitadoras não só no contexto académico, mas também na prática clínica.
\end{abstract}

Palavras-chave: Aprendizagem, Educação, Enfermagem, Estudantes, Tecnologias da Informação e Comunicação (TIC).

\section{WHAT IMPORTANCE DO NURSING STUDENTS ATTRIBUTE TO NEW TECHNOLOGIES}

Abstract. The development of science and technology introduced significant changes in the health area, constituting a challenge for the provision of nursing care. In the training of nurses, various types of educational equipment and technologies are used, with emphasis on information and communication technologies (ICT), whose objective is to facilitate the teaching-learning process, complementing traditional teaching methods. Nursing schools aim to prepare future nurses qualified to use the new emerging technologies and work in diverse contexts. In this article, it was intended to understand the importance that nursing students attach to ICT in the learning process. The methodology adopted was to conduct individual interviews with nursing students, for the treatment of the data was used content analysis. The results revealed that students recognize the advantages of ICT in their learning as future health professionals. They mainly use the computer and mobile phone with the aim of conducting research, communicating and conducting an autonomous study, which facilitates the teaching-learning process, decision making and the promotion of continuity in nursing care. In short, nursing students consider ICT as facilitating tools not only in the academic context, but also in clinical practice.

Keyword: Education, Information and Communication Technologies (ICT), Learning, Nursing, Students.

\section{INTRODUÇÃO}

Atualmente a saúde e as Tecnologias da Informação e Comunicação (TIC) são temas bem presentes no nosso quotidiano em diversos domínios do conhecimento. 
A compreensão da intercepção destas áreas implica reconhecer a saúde como tema de vital importância para a natureza humana (física, psicológica, social e espiritual) e sociedade constituída por ela e para ela. Por outro lado, a utilização das TIC na educação tem crescido rapidamente nas últimas décadas, nomeadamente no âmbito do ensino superior e, especificamente no âmbito do ensino em enfermagem (Button, Harrington, \& Belan, 2014), o que não só alterou a forma como vivemos e trabalhamos, mas também criou a necessidade de transformar o modo como aprendemos (Venkatesh, Bala, Sykes, \& Management, 2010).

A formação convencional em sala de aula deixou de ser o único espaço de aquisição e acesso ao conhecimento para adquirir e desenvolver novas aprendizagens. A internet permite comunicar e partilhar informações, pondo ao dispor saberes que podem ser transformados em conhecimento. As novas tecnologias permitem ainda a existência de ensino à distância em modo de e-learning e, deste modo o estudante passa a ter maior responsabilidade na sua aprendizagem e o professor como tutor, pode conduzir e apoiar o estudante a construir o conhecimento (Garrison, 2011). As ferramentas digitais à disposição nas instituições de ensino como as plataformas de partilha de documentos, possibilitam o ensino e-learning permitindo a comunicação estudante-professor à distância, complementando o método tradicional de ensino presencial (Woo \& Kimmick, 2000).

As escolas de enfermagem preparam os futuros enfermeiros para exercer a sua profissão em unidades de saúde cada vez melhor equipadas com tecnologias digitais. Assim, é esperado que tanto os professores de enfermagem, como os estudantes de enfermagem incorporem estas tecnologias precocemente para facilitar o processo ensino-aprendizagem e a integração no mundo do trabalho (Elder \& Koehn, 2009).

A utilização das TIC nos cuidados de enfermagem, permite aceder a conhecimento para fundamentar a tomada de decisões relacionadas com os cuidados de enfermagem, organizar os serviços de saúde relativamente a registos, avaliação e gestão dos cuidados prestados. Por outro lado, possibilita rentabilizar os recursos humanos e tecnológicos tornando os sistemas de saúde mais eficazes. A utilização de programas informatizados de registo dos cuidados de saúde utilizando linguagem uniformizada, veio permitir a criação de indicadores de saúde que refletem a qualidade da prestação dos cuidados de enfermagem (Lima, Antunes, \& Silva, 2015).

A facilidade com que se adquirem dispositivos eletrónicos móveis como computadores portáteis, tablets e telemóveis, bem como, o acesso rápido e fácil à internet, tornou possível 
aceder em qualquer hora e em qualquer lugar a redes sociais e a informação de acesso livre on-line. Esta realidade, mudou a forma como os estudantes aprendem e comunicam, nomeadamente os estudantes de enfermagem (Mariño, Habibi, Morgan, \& Au-Yeung, 2012). Estes contactam com as TIC não apenas nas instituições de ensino, mas também nos estágios realizados em hospitais e outras instituições de saúde e, estas são, portanto, uma importante ferramenta de aprendizagem e de trabalho como futuros enfermeiros.

Com o desenvolvimento da tecnologia surge também a e-saúde como uma realidade atual e intrínseca aos cuidados de saúde em todo o mundo, sendo um conceito que se baseia na capacidade das novas tecnologias, nomeadamente a internet, permitir elevar o conhecimento das pessoas sobre a sua saúde. A e-saúde possibilita também melhorar a gestão de cuidados e recursos de saúde, facilitar a comunicação entre os profissionais deste ramo e entre estes e as pessoas que deles necessitam, promovendo a maior acessibilidade aos cuidados (Shaw et al., 2017). Deste modo, as TIC são cada vez mais utilizadas nos cuidados de saúde, como forma de ajudar a pessoa com doença crónica ou aguda a gerir a sua condição. Existem vários estudos que comprovam a eficácia das intervenções web como forma de fornecer programas educacionais e monitorizar doenças crónicas (ex: diabetes e DPOC). Estes programas ajudam a promover o autocuidado e a autoeficácia dos doentes, tornando-os mais participativos na gestão da sua doença (Stellefson et al., 2013).

Como docentes de enfermagem numa Escola Superior de Enfermagem, consideramos que as TIC têm um papel fulcral no processo formativo dos estudantes, quer a nível teórico e teórico-prático, quer no âmbito do ensino clínico, sendo este último uma fase crucial na sua formação (Charleston \& Happell, 2005). Pensamos que o presente estudo encontra a sua fundamentação neste pressuposto, ambicionando compreender o processo de preparação dos nossos estudantes e de ser enfermeiro, bem como, explorar a importância que estes atribuem a estas tecnologias durante a sua formação em enfermagem, pretendendo contribuir para a melhoria contínua da formação dos estudantes de enfermagem.

O presente estudo tem como finalidade analisar a importância da utilização das TIC no processo ensino-aprendizagem, na perspetiva dos estudantes da Licenciatura de Enfermagem e, como pode contribuir para a melhoria do processo de ensino-aprendizagem.

Esboçada a finalidade é imprescindível o enunciado dos objetivos do estudo, os quais indicam a direção da investigação (Fortin, 2009). Assim, o principal objetivo deste estudo foi 
compreender que importância atribuem os estudantes de enfermagem às TIC no processo de ensino-aprendizagem durante o curso de enfermagem.

\section{METODOLOGIA}

Dada a problemática e o objectivo do estudo, optou-se por um estudo qualitativo exploratório e descritivo.

Relativamente aos participantes e, tendo em consideração a investigação de cariz qualitativo, estes foram selecionados de forma intencional. No presente estudo, a amostra é constituída por 12 estudantes de enfermagem do Curso de Licenciatura de uma Escola Superior de Enfermagem em Portugal.

A seleção do método para obter a informação constitui um aspeto importante do processo de investigação. Cabe ao investigador definir o tipo de instrumento, que melhor se adequa ao objetivo do estudo (Fortin, 2009). Uma das técnicas mais usadas, para a obtenção de dados em estudos qualitativos é a entrevista. As “(...) entrevistas são uma poderosa técnica de recolha de dados porque pressupõem uma interação entre o participante e o investigador, possibilitando a este último a obtenção de informação que nunca seria conseguida através de um questionário." (Silverman 2000, citado por Coutinho, 2014, p. 141). Existem diferentes variantes da entrevista, mas a mais correntemente utilizada é a semiestruturada ou semidirigida (Fortin, 2009; Quivy \& Campenhoudt, 2008). A entrevista semiestruturada é utilizada principalmente, quando o investigador pretende compreender, o significado de um acontecimento ou de uma experiência interpretada pelos participantes, onde estes expõem as suas ideias e percepções. Fortin (2009), considera que a entrevista semiestruturada possibilita uma interacção verbal que permite de forma flexível o investigador guiar a entrevista com o objectivo de abordar os temas sobre os quais deseja ouvir o participante, permitindo uma compreensão natural e autêntica do fenómeno em estudo (Fortin, 2009). O investigador geralmente dispõe de algumas questões pré-definidas, que podem ou não ser colocadas pela ordem ou pela formulação prevista (Quivy \& Campenhoudt, 2008).

Face ao exposto, como método de recolha de dados foi utilizada a entrevista semiestrututada, considerando-se ser o mais adequado face à finalidade deste estudo, constituindo-se como um método para obter informação espontânea e voluntária dos participantes (Bardin, 2011). 
A recolha de dados decorreu no período compreendido entre outubro e novembro de 2019. Para realizar as entrevistas, foi previamente pedida autorização à Comissão de Ética da instituição à qual os estudantes pertenciam e, também aos participantes, garantindo a confidencialidade de todas as informações, assim como, o anonimato.

Todas as entrevistas foram realizadas pelas investigadoras, tendo sido gravadas, em suporte áudio, para posterior transcrição e análise de conteúdo. Foram programadas de acordo com a disponibilidade e com a localização mais favorável a cada participante, em ambiente calmo. Estas tiveram a duração entre 30 a 60 minutos.

Para a análise dos dados foi escolhida a metodologia de análise de conteúdo, que de acordo com Bardin (2011) é importante pela necessidade de ultrapassar as incertezas face a algumas temáticas e descobrir o que se pretende investigar. Esta metodologia pode ser definida como um conjunto de instrumentos metodológicos, em constante aperfeiçoamento, que possibilita analisar diferentes fontes de conteúdos (verbais ou não-verbais).

A realização da análise dos dados abrange várias etapas, para conferir significados aos dados colhidos (Minayo, Deslandes, \& Gomes, 2001). Essas etapas são organizadas em três fases: 1) pré-análise, 2) exploração do material e 3) tratamento dos resultados, inferência e interpretação.

A primeira fase, pré-análise, permite sistematizar as ideias que emergem no quadro teórico e estabelecer indicadores para a interpretação da informação colhida. Esta fase compreende a leitura geral de todo o material para a análise, no caso de análise de entrevistas, estas devem ser transcritas. De forma geral, efectua-se a organização do material a ser investigado. Tal sistematização serve para que o investigador possa continuar as sucessivas fases da análise de conteúdo. Esta fase compreende a leitura flutuante que é o primeiro contacto que o investigador tem com os dados colhidos, momento em que se começa a conhecer o conteúdo das entrevistas a serem analisadas, o que constitui o corpus da análise e, ainda elaborar os indicadores a fim de interpretar o material.

Concluída a primeira fase, acima descrita, parte-se para a exploração do material, que constitui a segunda fase. A exploração do material consiste na construção de operações de codificação. Bardin (2011), define codificação como a transformação, por meio de excertos do texto, frases com agregação e enumeração, com base em regras precisas sobre as informações textuais, representativas das características do conteúdo. Nesta fase, o texto das 
entrevistas, é recortado em unidades de registro. Consideram-se unidades de registro os recortes dos textos (excertos, parágrafos, frases). Estas são identificadas, para realizar uma primeira categorização.

As primeiras categorias, são agrupadas de acordo com temas correspondentes, e dão origem às categorias iniciais. As categorias iniciais, são agrupadas tematicamente, originando as categorias intermediárias e, estas últimas também aglutinadas em função da ocorrência dos temas, resultam nas categorias finais. O texto das entrevistas recortado em unidades de registro, são agrupadas tematicamente nas categorias iniciais, intermediárias e finais, as quais possibilitam as inferências (Bardin, 2011). Neste processo indutivo ou inferencial, procura-se compreender o sentido da fala dos entrevistados, a definição de regras de contagem e a classificação e agregação das informações nas categorias simbólicas ou temáticas.

A formulação dessas categorias segue os princípios da exclusão mútua (entre categorias), da homogeneidade (dentro das categorias), da pertinência na mensagem transmitida (não distorção), e da objetividade (compreensão e clareza) (Bardin, 2011).

A terceira fase compreende o tratamento dos resultados, inferência e interpretação, consiste em interpretar os conteúdos manifestos e latentes contidos em todo o material (entrevistas). A análise comparativa é realizada através da justaposição das diversas categorias existentes em cada análise, ressaltando os aspetos considerados semelhantes e os que foram concebidos como diferentes.

É importante ressaltar que a análise dos dados a serem analisados obedece a princípios (Bardin, 2011) como:

- Exaustividade, significa que todos os dados são incluídos na análise e que os elementos não são deixados de fora sejam quais forem as razões.

- Representatividade, ou seja, a seleção dos dados deve ser rigorosa e ser representativa do universo das entrevistas.

- Homogeneidade os dados devem obedecer a critérios precisos de escolha e não podem fazer parte de várias categorias.

- Pertinência, significa que os dados correspondem ao objetivo que está a ser investigado, ou seja, esteja de acordo com o que se propõem no estudo. 
No presente estudo, na primeira fase ou pré-análise realizaram-se as entrevistas semiestruturadas orientadas por questões previamente elaboradas num protocolo, dando ao participante liberdade para responder espontaneamente, completando-as e enriquecendo-as durante a mesma. O guião da entrevista elaborado compreende cinco questões, tendo por base a literatura consultada e a experiência das investigadoras, como:

- Quais as TIC que usa mais a nível académico?

- O que representam para si as TIC, no processo ensino-aprendizagem/formação?

- Como considera que as TIC o/a podem ajudar a preparar-se como enfermeiro/a?

- Num futuro próximo, o que gostaria de ter à sua disposição em termos tecnológicos?

- Como é que as TIC o/a podem ajudar a cuidar de uma pessoa doente, em especial no caso de uma doença crónica?

Obteve-se uma grande quantidade de dados, os quais foram organizados e analisados através do programa MAXQDA 10. Foi realizada uma leitura flutuante necessária para trabalhar a informação, organizando-a e salientando o essencial, ou seja, foram optimizados os textos tornando os dados recolhidos mais compreensíveis, dando especial atenção aos princípios, como referido anteriormente.

$\mathrm{Na}$ segunda fase - exploração do material - considerámos que as questões colocadas constituem os respetivos temas, seguindo-se a etapa de agrupar as categorias. Para alguns dos temas, salientaram-se várias categorias finais e, para cada uma das categorias foram identificadas as respetivas unidades de registo.

A codificação corresponde a uma transformação dos dados, transformação esta, que sofreu muitos reajustes e aperfeiçoamentos, na tentativa de encontrar uma melhor compreensão para o conhecimento sobre a temática em estudo.

$\mathrm{Na}$ terceira fase - tratamento dos resultados, inferência e interpretação, elaborámos quadros que resumem a informação já processada em resultados que se traduzem em temas com as respetivas categorias, e estas com as respetivas unidades de registo e, a partir dos quais, interpretámos os resultados. 


\section{RESULTADOS/DISCUSSÃO}

Neste estudo participaram 12 estudantes, maioritariamente do género feminino $(n=8)$, com idades compreendidas entre os 18 e 23 anos (média de idades 19,3 anos). Destes, $78 \%$ eram estudantes do segundo ano e $22 \%$ do terceiro ano. Verificou-se que todos os estudantes (100\%) têm telemóveis e computadores com acesso à internet.

Quando se questionaram os estudantes “Quais as TIC mais utilizadas a nível académico?”, a maioria referiu que as mais utilizadas quer no seu dia-a-dia, quer nos seus estudos são o computador, telemóvel e tablet com acesso à internet. No entanto, alguns estudantes referem ainda outros recursos tecnológicos como as redes sociais, bases de dados e plataformas elearning. Estes resultados são também corroborados por outros autores como Deltsidou, Gesouli-Voltyraki, Mastrogiannis e Noula, 2010; Honey (2018) e Nguyen, Zierler e Nguyen (2011) que referem, nos seus estudos a utilização destas TIC pelos estudantes a nível académico e pessoal.

No tema "O que representam as TIC para si, no processo ensino-aprendizagem/ formação?" emergiram da análise de conteúdo, quatro categorias, nomeadamente: conhecimento académico; conhecimento geral e do mundo; realização de trabalhos académicos e ainda a sua utilização no lazer (Tabela 1). Globalmente os estudantes consideram que as TIC permitem aprofundar o conhecimento académico e científico e, também noutros domínios. Salientam que lhes permitem ter acesso ao conhecimento rápido, fácil e atual, podendo assim, realizar trabalhos solicitados pelos professores. Referem ainda que thes proporcionam momentos de lazer e relacionamento/interação à distância com os outros.

Os resultados desta investigação fornecem dados importantes sobre quais os TIC mais utilizadas pelos jovens estudantes e qual a sua finalidade. Conhecer estes aspetos, pode ter impacto nas decisões futuras de como a tecnologia é utilizada no ensino e aprendizagem, fornecendo informações importantes para subsidiar a prática docente nas Escolas de Enfermagem (Honey, 2018). Os resultados apontam ainda para o forte uso dos recursos online pelos alunos para acesso a informações sobre os conteúdos necessários e informações específicas, que são desenvolvidos a nível teórico e teórico-prático no curso, assim como, na realização de trabalhos individuais e de grupo.

Tabela 1. Distribuição das categorias, unidades de registo e enumeração relativamente ao tema "O que representam as TIC para si, no processo de ensino-aprendizagem/formação". 


\begin{tabular}{c|l|c}
\hline \multicolumn{1}{c|}{ Categorias } & \multicolumn{1}{c}{ Unidade de Registo } & \multicolumn{1}{c}{$\begin{array}{c}\text { Unidade de } \\
\text { Enumeração }\end{array}$} \\
\hline \multirow{5}{*}{ Conhecimento Académico } & Recolha de Informação & 12 \\
\cline { 2 - 3 } & Método de pesquisa & 6 \\
\cline { 2 - 3 } & Acessibilidade/Rápido acesso ao conhecimento & 5 \\
\cline { 2 - 3 } & Facilitador da aprendizagem & 1 \\
\cline { 2 - 3 } & Aprofundar conhecimentos & 1 \\
\cline { 2 - 3 } & Análise de Informação & 2 \\
\hline \multirow{4}{*}{ Conhecimento geral e do mundo } & Comunicação entre as pessoas e o mundo & 4 \\
\cline { 2 - 3 } & Desenvolvimento e formação pessoal & 2 \\
\cline { 2 - 3 } & Divulgar de informação & 5 \\
\cline { 2 - 3 } & Aceder a noticias do mundo & 2 \\
\hline \multirow{2}{*}{ Realização de Trabalho } & Facilitador na realização de trabalhos académicos & 3 \\
\cline { 2 - 3 } & Partilha de informação & 4 \\
\hline \multirow{2}{*}{ Lazer } & Jogos e música & 11 \\
\cline { 2 - 3 } & Utilização de Redes sociais & \\
\hline
\end{tabular}

Quando perguntámos aos estudantes "Como as TIC o podem ajudar a preparar-se para a sua futura profissão?" neste tema salientam-se duas categorias, que surgiram também da análise de conteúdo como: conhecimento técnico, científico e teórico e, ainda desenvolvimento de competências profissionais. Relativamente ao conhecimento os estudantes evidenciam que as TIC Ihes permitem realizar pesquisa atual, partilha de informação e estudo autónomo, sendo facilitador da aprendizagem e permitindo aceder a documentação pertinente para a prestação de cuidados mais eficazes. Os estudantes referem que o recurso a plataformas elearning pelas quais tem ao seu dispor conteúdos através de textos, vídeos e imagens, lhes facilita a apropriação de conhecimentos teóricos e técnico-científicos, fundamentais para a prática da enfermagem.

Por outro lado, a metodologia e-learning segundo estes, permite que possam, à distância, manter a comunicação entre estudante e professor através de casa, sendo este mais ativo e responsável pela sua aprendizagem (Button, Harrington, \& Belan, 2014). No que diz respeito ao desenvolvimento de competências, os estudantes destacam a atualização e aquisição de conhecimentos, assim como, ter acesso a evidência científica.

A maioria dos estudantes concorda que estas tecnologias aumentam o seu conhecimento no âmbito dos saberes em enfermagem, aumentando a confiança no estudo e habilidades clínicas, facilitando também a tomada de decisão relativamente aos cuidados a implementar no contexto da prática (O'Connor \& Andrews, 2018).

No que que diz respeito à questão "Em termos tecnológicos o que gostaria de ter à sua disposição num futuro próximo?", os estudantes referem que o mais importante é terem 
acesso a equipamentos de registo e de consulta móvel, com acesso à informação a nível das várias instituições de saúde. Também referem, que seria importante terem disponíveis softwares/plataformas de registo uniformizado em saúde que promovam a continuidade dos cuidados.

A linguagem de enfermagem padronizada facilita o encontro, processamento e gestão de uma enorme quantidade de dados e, a informação colabora para o progresso da prática clínica, pesquisa, educação e disseminação dos saberes em enfermagem (Silva, 2016). Alguns estudantes, fazem referência a outro tipo de softwares relacionados com a gestão de cuidados de saúde no âmbito da urgência e segurança do doente. Os estudantes consideram, que estas tecnologias são ferramentas que podem usar para aceder a recursos educacionais adequados durante a prática clínica.

A velocidade e acessibilidade com que a informação pode ser acedida são também consideradas como vantagens em comparação com os métodos mais tradicionais. No entanto, um estudante referiu que a obtenção de conhecimentos através das tecnologias não substituiu o treino em prática simulada ou a experiência do treino em contexto da prática clínica e, outros acreditam que o contato direto com o doente é a melhor maneira de aprender.

Alguns estudantes deram sugestões sobre como melhorar os aspetos pedagógicos destas aplicações, referindo que gostariam de ter mais informações audiovisuais, através de diagramas ou vídeos.

O tema "Como as TIC o podem ajudar a cuidar a pessoa doente/doença crónica?", surge com seis categorias, destacando-se a tomada de decisões de cuidados baseados na evidência, a continuidade de cuidados e a gestão da doença.

A implementação das TIC nas unidades de saúde, são apontadas como um meio para alcançar a qualidade dos cuidados em saúde, garantir a segurança do doente, reduzir o erro e diminuir os custos em saúde (Karimi, Poo, \& Tan, 2015). 
Tabela 2. Distribuição das categorias, unidades de registo e enumeração relativamente ao tema "Como as TIC o podem ajudar a cuidar a pessoa doente/doença crónica"

\begin{tabular}{|c|c|c|}
\hline Categorias & Unidades de Registo & $\begin{array}{l}\text { Unidade de } \\
\text { Enumeração }\end{array}$ \\
\hline \multirow{3}{*}{$\begin{array}{l}\text { Tomar decisões de } \\
\text { cuidados baseados } \\
\text { na evidência } \\
\text { científica }\end{array}$} & Adaptar os cuidados à pessoa de forma fundamentada & 10 \\
\hline & Individualização dos cuidados & 1 \\
\hline & Pesquisa de informação credível & 2 \\
\hline \multirow{3}{*}{$\begin{array}{l}\text { Continuidade de } \\
\text { cuidados }\end{array}$} & Registo rápido de dados & 3 \\
\hline & Acesso ao processo clínico do doente & 9 \\
\hline & Registo completo de dados do doente & 1 \\
\hline \multirow{8}{*}{ Gestão da doença } & Acompanhamento sintomas e terapêutica & 2 \\
\hline & Melhorar comunicação enfermeiro-doente & 2 \\
\hline & Vigilância dos sintomas & 2 \\
\hline & Follow-up à distância & 1 \\
\hline & Registo das queixas do doente & 1 \\
\hline & Informação sobre grupos de apoio e ajuda & 1 \\
\hline & Equipamentos de gestão de sintomas e melhoria da qualidade de vida & 1 \\
\hline & Promover a autonomia & 1 \\
\hline \multirow{2}{*}{ Promover o conforto } & Pesquisa dos produtos de apoio mais ajustados ao doente & 1 \\
\hline & Identificar medidas de alívio e conforto & 1 \\
\hline Controlo da dor & Monitorizar necessidades do doente & 1 \\
\hline \multirow{2}{*}{$\begin{array}{l}\text { Acesso aos } \\
\text { cuidados }\end{array}$} & Encaminhamento para unidades de saúde & 1 \\
\hline & Informação de contactos das instituições de saúde & 1 \\
\hline
\end{tabular}

Os estudantes consideram, que a evidência fornece fundamentos para a tomada de decisão através dos resultados de pesquisas desenvolvidos em investigações realizadas por especialistas reconhecidos da área (Galvão, Sawada, \& Rossi, 2002), contribuindo assim para a qualidade dos cuidados. Os estudantes valorizam neste âmbito os sistemas de registo e consulta dos processos clínicos dos doentes, sendo fundamentais para os conhecer e promover a continuidade e efetividade dos cuidados.

Sobressaiu também na opinião dos mesmos, o facto destas ferramentas serem importantes para a gestão da doença, nomeadamente através de aplicações que permitam a comunicação enfermeiro-doente. Estas fornecem a informação necessária ao próprio e família, aumentando a adesão de comportamentos associados à saúde e estilos de vida, conhecimentos e informações sobre o controle dos sintomas, logo permitem uma melhor autogestão da sua condição, diminuindo as visitas ao hospital e, melhor adesão ao regime terapêutico. Existe neste âmbito evidência que as TIC têm efeitos positivos em pessoas com doenças crónicas, nomeadamente na promoção da autocuidado e, de autogestão da sua saúde (Sá, Oliveira \& Nabais, 2018). De acordo com Cowie et al. (2016), a e-saúde tem o potencial de fornecer soluções inovadoras para os problemas de saúde da população, que se encontra cada vez mais familiarizada e habilitada para a utilização das TIC. As pessoas esperam das novas tecnologias formas aceder a partir de qualquer sítio à informação e a cuidados de saúde mais 
personalizados. Nesta categoria são citadas com menor evidência o controlo da dor e promoção do conforto, estes constituem uma preocupação dos estudantes como resultado da intervenção do enfermeiro à pessoa com doença crónica proporcionando assim, o seu bemestar (Sá, Oliveira \& Nabais, 2018).

\section{CONCLUSÕES}

O rápido desenvolvimento das TIC apresenta um enorme impacto no processo ensinoaprendizagem dos estudantes de enfermagem. Estas, não só permitem a aquisição de conhecimento, mas também constituem ferramentas importantes para o trabalho futuro como profissional de enfermagem, uma vez que a tecnologia emergente contribui para novas possibilidades de formação profissional, tendo também grande importância no processo de melhoria da qualidade de cuidados (Oliveira et al., 2014) e, consequentemente ganhos em saúde. Neste sentido, facilitar o acesso à internet e promover a literacia digital torna-se fundamental no presente e futuro para que possa ser alcançado um sistema de saúde equitativo e centrado na pessoa/doente.

Este estudo evidencia que os estudantes consideram as TIC relevantes na sua formação em contexto formal ou académico para aprofundar e adquirir conhecimento, facilitando a realização de trabalhos individuais e em grupo. Na formação em contexto clínico permite a tomada de decisão com base em evidência científica atual. Por outro lado, estes consideram que as novas tecnologias constituem um meio rápido e eficaz para facilitar a comunicação à distância entre as pessoas e profissionais de saúde, contribuindo para uma melhor gestão da saúde, especialmente no caso das doenças crónicas.

Para haver desenvolvimento de competências profissionais deverá aprofundar se o conhecimento e, para isso é necessário adotar processos de investigação e prática baseada na evidência de cariz estruturante para a profissão, contribuindo em grande medida as tecnologias. Este estudo fornece informações úteis, sobre quais as TIC que os estudantes mais utilizam e valorizam na aquisição de competências como futuros enfermeiros. As TIC fornecem uma metodologia útil e de baixo custo para o desenvolvimento de intervenção educativa em enfermagem, tornando mais eficaz e rigoroso o processo de ensinoaprendizagem. Este conhecimento, pode ter impacto nas decisões futuras sobre as tecnologias que são usadas no ensino das Escolas de Enfermagem. 
Os resultados sugerem ainda, que este estudo pode servir de ponto de partida para outros mais aprofundados e, que avaliem o impacto, a eficácia e as perceções de estudantes e professores que utilizam as TIC para melhor decidir sobre o futuro do ensino em enfermagem.

Face aos resultados obtidos, podemos inferir que as Escolas de Enfermagem não podem alhear-se do que se passa à sua volta e devem utilizar os recursos disponíveis de forma a contribuir para a formação de futuros enfermeiros aptos para os desafios da saúde, da tecnologia, da sociedade, da informação e conhecimento. Para tal, é necessário, e importante uma conscientização por parte dos professores de enfermagem da importância das TIC no processo de ensino-aprendizagem, assim como, o seu treino e o seu adequado envolvimento.

\section{REFERÊNCIAS}

Bardin, L. (2011). Análise de conteúdo. São Paulo: Edições 70.

Button, D., Harrington, A., \& Belan, I. (2014). E-learning \& information communication technology (ICT) in nursing education: A review of the literature. Nurse Education Today, 34(10), 1311-1323. doi:https://doi.org/10.1016/j.nedt.2013.05.002.

Charleston, R. \& Happell, B. (2005). Psychiatric nurses and undergraduate nursing students` perceptions of preceptorship in the mental health setting. The international Journal of Psychiatric Nursing Research,10(3), 1543 - 1549.

Coutinho, C. (2014). Metodologia de Investigação em Ciências Sociais e Humanas: Teoria e Prática. Coimbra: Almedina.

Cowie, M. R., Bax, J., Bruining, N., Cleland, J. G., Koehler, F., Malik, M., ... \& Vardas, P. (2016). e-Health: a position statement of the European Society of Cardiology. European heart journal, 37(1), 63.

Deltsidou, A., Gesouli-Voltyraki, E., Mastrogiannis, D., \& Noula, M. (2010). Undergraduate nursing students' computer skills assessment: a study in Greece. Health Science Journal, 4 (3), 182.

Elder, B., \& Koehn, M. (2009). Assessment ToolforNURSING STUDENTComputer Competencies. Nursing Education Perspectives, 30(3), 148-152.

Fortin, M. (2009). Fundamentos e etapas do processo de investigação. Loures: Lusodidacta.

Galvão, C., Sawada, N, \& Rossi, L. (2002). A prática baseada em evidências: considerações teóricas para sua implementação na enfermagem perioperatória. Revista Latino-Americana de Enfermagem, 10(5), 690-695.

Garrison, D. R. (2011). E-learning in the 21st century: A framework for research and practice. London: Routledge.

Honey, M. (2018). Undergraduate Student Nurses' Use of Information and Communication Technology in Their Education. Studies in health technology and informatics, 250, 37-40.

Karimi, F., Poo, D, \& Tan, Y. (2015). Clinical information systems end user satisfaction: the expectations and needs congruencies effects. Journal of biomedical informatics, 53, 342-354.

Lima, K., Antunes, J., \& Silva, Z. (2015). Percepção dos gestores sobre o uso de indicadores nos serviços de saúde. Saúde e Sociedade, 24, 61-71. 
Mariño, R., Habibi, E., Morgan, M., \& Au-Yeung, W. (2012). Information and communication technology use among Victorian and South Australian oral health professions students. Journal of Dental Education, 76(12), 16671674.

Minayo, M., Deslandes, S., \& Gomes, R. (2011). Pesquisa social: teoria, método e criatividade. Editora Vozes Limitada.

Nguyen, D., Zierler, B., \& Nguyen, H. (2011). A survey of nursing faculty needs for training in use of new technologies for education and practice. Journal of nursing education, 50(4), 181-189.

O'Connor, S., \& Andrews, T. (2018). Smartphones and mobile applications (apps) in clinical nursing education: A student perspective. Nurse Education Today, 69, 172-178.

Oliveira, D., Melo, V., Duarte, E., Sá, S., Andrade, M., \& Cavalcanti, A. (2014). Atuação do tutor no ensino superior a distância: revisão integrativa. Revista de Enfermagem UFPN, 8(4), 1029-1037.

Quivy, R.; Campenhoudt, L. (2008). Manual de Investigação em Ciências Sociais. (5ª ed). Lisboa: Gradiva.

Sá, C., Oliveira, A., \& Nabais, A. (2018). The importance of Information and Communication Technologies (ICT) to promote Information, self-care and empowerment among patients with chronic illnesses. In L. Gómez Chova, A. López Martínez, \& I. Candel Torres (Eds.), 10th International Conference on Education and New Learning Technologies, EDULEARN18 PROCEEDINGS. (pp. 7993-7998). Palma de Maiorca: IATED Academy. DOI: 10.21125/edulearn.2018.1858. Disponível em: http://hdl.handle.net/10071/17002.

Shaw, T., McGregor, D., Brunner, M., Keep, M., Janssen, A., \& Barnet, S. (2017). What is eHealth (6)? Development of a conceptual model for eHealth: qualitative study with key informants. Journal of medical Internet research, 19(10), e324.

Silva, P. S. (2016). Satisfação dos enfermeiros na utilização de sistemas de informação em enfermagem: Um estudo nos hospitais do Funchal. (Dissertação de Mestrado), ESEP, Porto.

Stellefson, M., Chaney, B., Barry, A., Chavarria, E., Tennant, B., Walsh-Childers, K., . . Zagora, J.(2013). Web 2.0 chronic disease self-management for older adults: a systematic review. Journal of Medical Internet Research, 15(2), e35.

Venkatesh, V., Bala, H., Sykes, T., \& Management, O. (2010). Impacts of information and communication technology implementations on employees' jobs in service organizations in India: a multi-method longitudinal field study. Production and Operations Management, 19(5), 591-613.

Woo, M., \& Kimmick, J. (2000). Comparison of Internet versus lecture instructional methods for teaching nursing research. Journal of Professional Nursing, 16(3), 132-139. 